\title{
Evaluating Cost Allocation Imposed by Cooperative Switching Off in Multi-Operator Shared HetNets
}

\author{
Maria Oikonomakou, Student Member, IEEE, Angelos Antonopoulos, Senior Member, IEEE, Luis Alonso, Senior \\ Member, IEEE and Christos Verikoukis, Senior Member, IEEE
}

\begin{abstract}
The anticipated explosion in the total data traffic load will impose to mobile network operators (MNOs) the necessity to densify their networks to provide coverage. At the same time, since MNOs plan their networks according to their high-peak traffic load, base station underutilization during the low traffic hours raises the issue of unnecessary power consumption and excessive cost. In the present paper, we plan to study the energy and cost efficiency of a heterogeneous network (HetNet) that is a cooperation result of many MNOs. Each MNO is owner of a HetNet, composed of eNodeBs (eNBs) and smal cells (SCs) and they cooperate by sharing their infrastructure and by switching off a part of it. BS type and traffic load constitute switching off criteria and a roaming cost based user association scheme is used to roam traffic to neighbouring BSs. We assess the cost alterations created by the possible MNO coalitions and we propose a bankruptcy game to allocate the obtained cost to the cooperative MNOs and to motivate thus them to maintain their sharing agreement instead of following a non-cooperative tactic. The bankruptcy game uses Shapley Value to portray each MNO's contribution to cost savings. The MNOs' satisfaction from their payoffs (i.e., the allocated cost) and the overall fairness of the method are evaluated. According to the extracted results, the proposed switching off scheme achieves significant improvement of energy efficiency for the studied network, while the proposed bankruptcy game achieves a balanced and satisfactory cost allocation for different MNO traffic loads.
\end{abstract}

Index Terms-Switching off, network sharing, cost allocation, bankruptcy theory, Shapley Value.

\section{INTRODUCTION}

A huge augmentation of the mobile-connected devices per capita is expected by the end of 2021 according to recent studies (about 1.5 mobile-connected devices per capita) [1]. Mobile network operators (MNOs) will thus have to be able to live up to the demands of their subscribed user equipment devices (UEs) and cover their traffic. Triggered by their need to increase their network capacity, MNOs have embraced heterogeneous networks (HetNets), which allow the addition of extra base stations (BSs), such as macrocell BSs (MBSs), or eNodeBs (eNBs) for the Long Term Evolution Advanced (LTE-A) networks and small cells (SCs). However, capacity

M. Oikonomakou is with Iquadrat Informatica, Barcelona, Spain and with the Department of Signal Theory and Communications (TSC) of the Technical University of Catalonia (UPC), Barcelona, Spain, e-mail: (moikonomakou@iquadrat.com)

A. Antonopoulos and C.Verikoukis are with Telecommunications Technological Center of Catalonia (CTTC), Barcelona, Spain, e-mail:( (aantonopoulos, cveri)@cttc.es)

L. Alonso is with the Department of Signal Theory and Communications (TSC) of the Technical University of Catalonia (UPC), Barcelona, Spain, email: (luisg@tsc.upc.edu) and coverage augmentation through installation of extra infrastructure would oblige MNOs to confront two raising issues: energy and cost efficiency of their network.

On one hand, network densification is related to increased needs of electrical energy for its operation and thus to higher $\mathrm{CO}_{2}$ emissions. It has been estimated that information and communication technology (ICT) infrastructure is responsible for the $2 \%$ and $3 \%$ of total carbon dioxide $\left(\mathrm{CO}_{2}\right)$ emissions and power consumption, respectively. Increased power needs are translated into an electricity bill of more than 10 million $€$ [2], with BSs contributing up to $60-80 \%$ to the total energy consumption. Thus, a greener approach of network operation is projected as a necessity that cannot be neglected when reduced network capital and operational expenses (CapEx, OpEx) and lower $\mathrm{CO}_{2}$ emissions are set as aims.

On the other hand, MNOs need to reassure that the business model they follow for the operation of their network results in positive revenues. A dense single-handled network can prove to be very challenging financially for the owner MNO given the increasing traffic volume, while recently MNOs noticed for the first time a decrease in their revenues [3]. Recent researches, e.g., [4] and [5], indicate the tendency of industry operators to act against this phenomenon and proceed to network sharing agreements of different types, such as passive, active and roaming-based sharing [6]. Thus, they enable key drivers of their interest, i.e., reduction of the cost attributed to additional capacity and coverage by sharing bills [7].

Even though a viable network sharing agreement needs to result in energy and cost savings for the shared network, it also presumes a successful and fair cooperation among MNOs. Being already reluctant to refrain from the traditional standalone business model of managing only their own traffic, MNOs would not proceed to the new business model of sharing agreements unless it resulted in $(a)$ higher profit compared to the stand-alone one and $(b)$ fair treatment among cooperative MNOs.

Considering all the above, infrastructure switching off and sharing have been embraced as approaches that can contribute to both energy and cost saving. Given that networks are planned to serve traffic demands during the high-peak traffic hours, the phenomenon of underutilized infrastructure during low-traffic hours has arisen and been further aggravated with the adoption of dense HetNets. A HetNet could lead to considerable energy and cost savings, since it is composed of not only high power consuming ( 1.35 KW [8]) eNBs but also numerous SCs. Due to their big number, SCs can create a significant aggregated amount of power consumption, despite 
the fact that an individual SC is less power consuming than an eNB. As far as infrastructure sharing is concerned, hyper-dense HetNets in urban populated areas can lead to a BS instalment in close proximity due to scarcity of available space [9]. Thus, it can only motivate MNOs to share their infrastructure and their operational expenses (OpEx). For the owner MNOs that see their revenues progressively decreasing from the adoption of the individual operation of their network, such a movement could be a sigh of relief for their expenses [3]. For the success of an infrastructure sharing though, there needs to be sincerity by the cooperative MNOs. Apart from realising and acknowledging the economic benefits of sharing, an incentivebased policy must be put in place, as a way of encouraging and growing the culture of infrastructure sharing on a level playing field. To that end, cost allocation methods can be used to fairly allocate the costs among the members of a group. An appropriate cost allocation ensures less disgruntlement to the MNOs, especially if it is representative of their individual contribution to any cost saving achieved by their cooperation. Such a strategy would allow them to still have control of their investments and maintain their growth strategies.

With regard to the existing related work, significant research has been devoted to BS switching-off algorithms in both single-operator networks ( [10]-[13]) and multi-operator networks, where concerns about cost and revenue issues for network sharing are raised [14]-[18]. However, there are still open issues that should be given further consideration when it comes to the adoption of BS switching off in a multi-operator shared HetNet, as well as to the application of a cost allocation method. Energy savings should be achieved while taking into consideration the kind of infrastructure that should be switched off, i.e., eNB or SCs, as well as the network to which the infrastructure belongs. Moreover, the employed network sharing, if there is any, should be given consideration in the switching off strategies as it can strongly affect the profitability of a possible MNO coalition. Furthermore, a cost allocation has to address efficiently fairness issues among the MNOs. Even though the validity of a coalition and the profitability of each MNO itself are dependent on the fairness of an allocation of the network expenses, fairness issues have been given slight consideration so far on issues such as resource allocation [19].

The contribution of this paper is twofold:

- We study the low-traffic hour energy and cost efficiency of an extended HetNet, which is a collaboration result of multiple MNOs whose individual HetNets are located in a certain area. More specifically, we describe the cooperation of the MNOs as an application of the switching off technique to the eNBs and SCs of their roaming-based shared HetNets. We adopt a form of roaming-based sharing [6], according to which an MNO can roam its traffic to a rival MNO during a pre-defined period of time over a strictly pre-defined area. We formulate an energy efficient optimization problem and propose a cooperative greedy heuristic algorithm, namely Cooperative Switching Off (CSO), for its solution. We consider the type and load of a BS as criteria for the BS switching off selection and we propose a cooperative roaming-based UE association (UA) scheme to define the operation states of eNBs and
SCs. CSO has the benefits of being $(i)$ conceptually rather simple, (ii) easy to implement, and (iii) efficient so as to encounter a configuration of BS operation and UE association states that improves energy efficiency of the extended HetNet meanwhile capturing cooperation issues.

- Regarding the cost issues raised from a collaboration agreement that includes CSO, we model the cooperation and cost sharing decisions among MNOs using a Shapley Value based bankruptcy game (BSV). The bankruptcy game represents the idea of allocating a specific entity among a group of players who are interested in it [20][22]. In the present case, we use it to estimate the saved cost achieved from different coalition decisions among MNOs, in comparison to a non-cooperative network activity. Shapley Value (SV), thanks to the fairness characteristic it has as a method, is used to determine the individual contribution of each MNO to the total cost savings that eventually defines its allocated cost, in combination with its respective roaming revenues. The proposed scheme is also evaluated with respect to the fairness issues created among MNOs.

The remainder of this paper is organized as follows. Section II briefly reviews the related work. The system model, network configuration and notation followed throughout the paper are presented in Section III. In Section IV, we describe the proposed switching off scheme, while Section V analyses the coalitional bankruptcy game. A performance assessment of our proposals are provided in Section V. Finally, Section VII concludes the paper.

\section{RELATED WORK}

Since network infrastructure is the most power consuming part of the network, extensive work has already been devoted to energy saving strategies, such as [23] and [24]. Switching off algorithms have been presented towards this effort for both single- and multi-operator networks. Indicative examples for a single-operator HetNet are [10], where open-access femtocells are switched $\mathrm{ON}$ and $\mathrm{OFF}$ dynamically provided that a power saving UA scheme can reassure the service of UEs and [11], where a distributed graph based game that enables BSs to optimise their switching strategies for energy saving meanwhile guaranteeing the minimum service of their UEs is proposed. In addition, [12] presents a different switching off procedure that is applied to SCs only, depending on the UE distribution within each SC area. In detail, when UE distribution within each SC area is $(a)$ uniform, SCs are switched off dynamically according to their distance from the central eNB and $(b)$ nonuniform, SCs are switched off according to power saving lists that are formatted based on a power saving efficiency indicator $Q$. In both cases, SC deactivation is applied with traffic offloading to the eNB and until either no further improvement of the HetNet power consumption can be achieved or the eNB has reached its capacity or power limitations. Two sleeping schemes are also presented and compared in [13]: (a) a random one and (b) a traffic load based one, with the traffic load being counted as the number of the nearest to the BS UEs. A common characteristic of the aforementioned works is their focus on the dynamic BS operation by a single operator. 
Our work sets the focus on multi-operator scenarios, where infrastructure sharing can be adopted. Towards this direction, the authors of [14] and [15] study the relations between multiple MNOs and a third party. More specifically, in [14], they propose a non-cooperative auction-based game that aims at MNOs switching off their eNBs by totally offloading the eNB traffic to leased from a third party SCs, and in [15], they refer to cost sharing policies with reference to a third party. Other works consider only multi-operator scenarios. In detail, [16] discusses the deployment of extra SCs by cooperative MNOs based on the achievable UE throughput and the individual MNO revenues, as a result of their respective investments and the payments of their UEs. Moreover, [17] refers to a budget-balanced mechanism designed for MNOs of cellular networks with similar load distributions, while in [18] cellular network operators switch off their networks in a non-cooperative manner, aiming at energy efficiency. However, works of [17], [18] do not consider a HetNet scenario, which is a characteristic that could differentiate the results. Moreover, their discussion on the revenues issues is based on payment only agreements, without considering pricing differentiations among different types of BSs or cost sharing methods. Unlike the aforementioned works, in our work, we study purely MNO-dominated scenarios, providing a switching off solution that incorporates multiple network characteristics, such as traffic load, BS type and variable roaming charges, while we provide a novel fair cost sharing solution that reassures the profitable network operation.

\section{SySTEM MODEL}

In the present section, we will describe the system model on which we based our study and the technical details we considered for the network configuration, as well. The section also serves as an explicit guide for the basic notation we follow in the rest of the paper.

\section{A. Network System Model and Operation}

We study a densely populated macrocell-sized urban area $A$, where multiple MNOs, indexed by $n, n \in \mathcal{N} \triangleq\{1, . .,|\mathcal{N}|\}$, have located their LTE-A HetNets, as in Fig. 1. We assume that each $n \in \mathcal{N}$ is owner of a HetNet, composed of one eNB, located in the centre of $A$ and uniformly distributed small cells (SCs) in the rest of it. Let $m^{n}$ be an eNB $m$ of MNO $n, m^{n} \in \mathcal{M}^{n} \triangleq\left\{1, . .,\left|\mathcal{M}^{|N|}\right|\right\}$ and $s^{n}$ a SC $s$ of MNO $n, s^{n} \in \mathcal{S}^{n} \triangleq\left\{1, . .,\left|\mathcal{S}^{|N|}\right|\right\}$. Thus, a HetNet $\mathcal{L}^{n}=\left\{1, \ldots,\left|\mathcal{M}^{|N|}\right|,\left|\mathcal{M}^{|N|}\right|+1, . .,\left|\mathcal{M}^{|N|}\right|+\left|\mathcal{S}^{|N|}\right|\right\}$ is composed for each $n$, which we index with $l^{n}$. In order to facilitate notation in cases irrespective of the owner MNO $n$, we index with $m$ an eNB of all eNBs in $A, m \in \mathcal{M}=\mathcal{M}^{1} \cup . . \cup \mathcal{M}^{|N|}$ and a $\mathrm{SC}$ of all SCs in $A$ with $s, s \in \mathcal{S}=\mathcal{S}^{1} \cup \ldots \cup \mathcal{S}^{|N|}$. Finally, let $l, l \in \mathcal{L}=\mathcal{M} \cup \mathcal{S}=\{1, . .,|\mathcal{M}|, . .,|\mathcal{M}|+|\mathcal{S}|\}$, be a BS of the unified infrastructure, irrespective of both BS type (eNB or SC) and owner MNO.

We moreover assume that each $n \in \mathcal{N}$ is the service provider MNO for a group of UEs that are uniformly distributed in the studied area. If $K^{n}(t)$ is the expected number of MNO $n$ for the duration of hour $t$, then let $k^{n}$ be a UE $k$ of

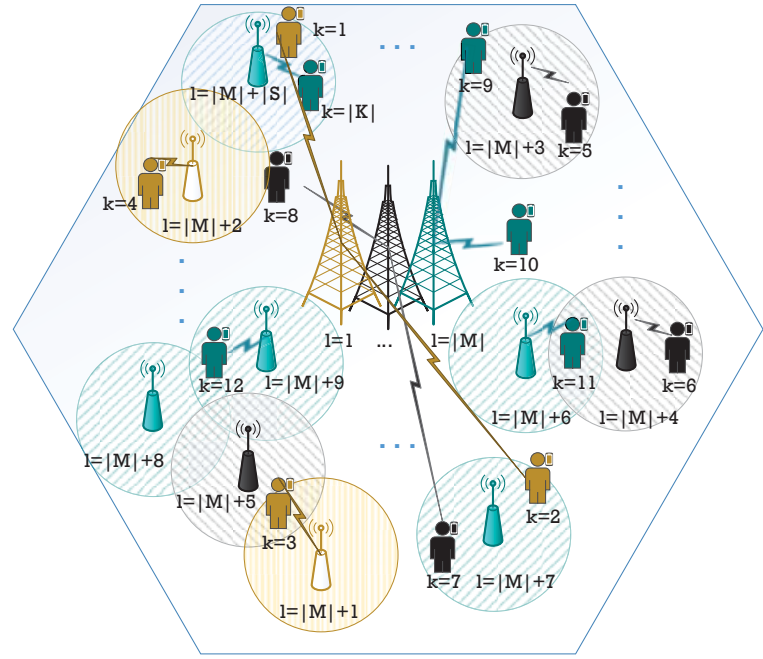

Figure 1. Studied macrocell-sized area $A$, where $\mathcal{N}$ MNOs have located and share their HetNets. The shared HetNet of $\mathcal{N}$ consists of $\mathcal{L}$ BSs in total (with $l \in \mathcal{L}$ ), $|\mathcal{M}|$ eNBs and $|\mathcal{S}| \mathrm{SCs}$ and it provides service to the total set of the subscribed UEs, $\mathcal{K}$ (with $k \in \mathcal{K}$ ).

MNO $n, k^{n} \in \mathcal{K}^{n}(t) \triangleq\left\{1, . .,\left|\mathcal{K}^{n}(t)\right|\right\}$. Under the roamingto-all assumption among the MNOs, we represent the total group of UEs that has to be served from all $\mathcal{N}$ cooperative MNOs with $\mathcal{K}(t)=\mathcal{K}^{1}(t) \cup \mathcal{K}^{2}(t) \cup \ldots \cup \mathcal{K}^{n}(t)$. Each UE $k \in \mathcal{K}(t)$ has a specific guaranteed bit rate demand, denoted by $\rho_{k}(t)$. We assume that there are $|\mathcal{I}|$ different categories of data throughput demands $r_{i}, i \in \mathcal{I}=\{1,2, . .,|\mathcal{I}|\}$. If $g(i)$ is the random probability with which a data throughput demand $r_{i}$ can be corresponded to a UE, with $g_{i} \in[0,1]$ and $g_{1}+. .+g_{i}+. .+g_{|\mathcal{I}|}=1$, then, $\rho_{k}(t)$ may be equal to $r_{i}$ with a random probability $g_{k, i}(t) \in\left\{g_{1}, g_{2}, . ., g_{i}\right\}$ during $t$. It is noted that, in the general case, $|\mathcal{I}|$ may be equal to $|\mathcal{K}(t)|$, as each $k$ may have different throughput needs.

Each of the UEs can initially get associated to and served by only one BS $l \in \mathcal{L}$, from which it receives the best signal-tonoise ratio (SNR). We denote the associate state of each $k$ with an $l$ during $t$ as $q_{k, l}(t)$, which is equal to 1 when $k$ is associated to $l$ and 0 otherwise. Each MNO is assumed to operate in a different frequency to avoid interference issues for its network [25]. Also, orthogonal transmission is adopted to avoid intracell interference, while inter-cell interference is considered to be mitigated through some form of fractional frequency reuse scheme or sophisticated frequency allocation [26].

We assume a form of roaming-based sharing [6], according to which UEs of different MNOs can be served by the network of other than their subscriber MNO in a certain area, for a predefined period of time and at the expense of set inter-operator charges. Each re-association event of a $k$ to an $l$ is considered unique and charged by the host $n$ with a price, $c$. It is noted that each $n$ is able to define a different $c$ in order to serve the traffic of other MNOs. In the present paper, we propose a roaming pricing model described in Section IV-C.

\section{B. BS Power Consumption Model}

One of our main interests is power consumption in $A$. Consequently, we focus on network power consumption attributed 
to $\mathrm{BSs}$, as a result of its signalling processes, cooling, battery needs and transmission activity. The downlink (DL) case is considered only, where orthogonal frequency division multiple access (OFDMA) scheme is employed.

The power consumption of a BS $l$ at $t$ is calculated as [27]

$$
P_{l}(t)=\theta_{l}(t) \cdot\left(P_{l}^{c o n}+\Delta P_{l} \cdot P_{l}^{t x}(t)\right),
$$

where $P_{l}^{c o n}$ is the constant power consumption of $l$ attributed to signal processing, battery backup and cooling. $\Delta P_{l}$ stands for the variable that scales the power consumption of $l$ with the radiated power, due to amplifier and feeder losses and $P_{l}^{t x}$ refers to the transmit power of $l$. It is noted that $P_{l}^{\text {con }}$ and $\Delta P_{l}$ take different values when $l$ refers to an eNB or SC, which are given later in the paper. Finally, each $l \in \mathcal{L}$, has two possible operation modes $\theta_{l}(t)$ during $t$ : (i) active, which corresponds to $\theta_{l}(t)=1$ and (ii) inactive, which corresponds to $\theta_{l}(t)=0$.

Thus, the power consumption of the roaming-based shared HetNet $\mathcal{L}$ of all $\mathcal{N}$ at $t$ is

$$
P^{\mathcal{L}}(t)=\sum_{l=1}^{l=|\mathcal{L}|} \theta_{l}(t) \cdot\left(P_{l}^{c o n}+\Delta P_{l} \cdot P_{l}^{t x}(t)\right) .
$$

\section{Channel Model}

The power that a BS needs for its transmission activities, $P_{l}^{t x}$, is related to the number of its associate UEs and their respective bit rate demands. Since we study the DL case of an OFDMA scheme, we assume that information is transmitted in pairs of resource blocks (RBs), with each RB being of $0.5 \mathrm{~ms}$ duration in the time domain. We calculate $P_{l}^{t x}(t)$ of an $l$ as the sum of power that corresponds to the total number of RBs that $l$ has allocated to associate UEs during $t$. Denoting with $J_{l}(t)$ the allocated RBs of $l$, or the traffic load of $l$ as we will call it hereafter, during $t$, it is

$$
J_{l}(t)=\sum_{k \in \mathcal{K}(t)} q_{k, l}(t) \cdot j_{k, l}(t),
$$

which results in a $P_{l}^{t x}$ calculated as

$$
P_{l}^{t x}(t)=J_{l}(t) \cdot P_{l}^{R B} .
$$

In eq. (3), $q_{k, l}(t)$ denotes the associate state of a $k$ with $l$ at $t$, $j_{k, l}(t)$ is the number of RBs that $l$ has to transmit to $k$ at $t$ in order to provide it with the service it has requested (i.e., the $\rho_{k}$ that $k$ demands). In eq. (4), $P_{l}^{R B}$ is the power consumption for the transmission of $1 \mathrm{RB}$ from BS $l$.

The quality of the channel between a $k$ and an $l$ and, consequently, the estimated SNR of the link, $S N R_{k, l}(t)$ eventually define $j_{k, l}(t)$ as [26]

$$
j_{k, l}(t)=\left\lceil\frac{\rho_{k}(t)}{W_{l}^{R B} f\left(S N R_{k, l}(t)\right)}\right\rceil,
$$

where $W_{l}^{R B}$ is the bandwidth that corresponds to an RB pair of $l$ and $f\left(S N R_{k, l}(t)\right)$ is the spectral efficiency of the link between $k$ and $l$ at $t$. We remind that $\rho_{k}(t)$ represents the guaranteed bit rate of $k$ during $t$.

In order to calculate $f\left(S N R_{k, l}(t)\right)$, we first calculate the $S N R_{k, l}(t)$ as follows [26]

$S N R_{k, l}(t)=P_{l}^{t x, s u b}+G_{l}^{t x}-P L_{k, l}(t)-F L_{k, l}-N_{t h}-N F$, where $P_{l}^{t x, s u b}$ represents the allocated power to each subcarrier of a BS $l(\mathrm{dBm}), G_{l}^{t x}$ denotes the antenna gain (including feeder loss, $d B i), P L_{k, l}(t)$ is the pathloss between $k$ and $l$ at $t(d B), F L_{k, l}$ denotes the slow fading losses $(d B), N_{t h}$ is the thermal noise and $N F$ is the noise figure. Moreover, we adopt the adaptive modulation and coding scheme (AMC) over any radio link. Consequently, the appropriate $S N R_{k, l}(t)$ will eventually define the modulation and coding scheme (MCS) that will be used over the link. More specifically, QPSK, 16QAM and 64QAM modulation schemes of different respective coding rates are considered. The mapping between requested $\rho_{k}(t)$ and $S N R_{k, l}(t)$ to the achievable $f\left(S N R_{k, l}(t)\right)$ is executed as indicated in [21, Table A.2] [28].

As far as $P_{l}^{t x, s u b}$ is concerned, having assumed that the transmit power of an $l, P_{l}^{t x}$, is equally distributed among its subcarriers, $P_{l}^{t x, s u b}$ is defined as

$$
P_{l}^{t x, s u b}=\frac{P_{l}^{t x, \max }}{12 \cdot h_{l} \cdot J_{l, \max }},
$$

where $P_{l}^{t r, \max }$ is the maximum transmit power of $l, h_{l}$ stands for the number of antennas of $l$ and $J_{l, \max }$ stands for the maximum capacity of $l$, i.e. the total number of RBs that $l$ has available for allocation. Based on this, we can calculate $P_{l}^{R B}$ of eq. (4), as

$$
P_{l}^{R B}=\frac{P_{l}^{t x, \max }}{h_{l} \cdot J_{l, \max }} .
$$

\section{ENERGY EFFICIENCY PROBLEM}

An aim of the present work is to increase the network energy efficiency by adopting both methods of switching off infrastructure and network sharing. The switching off method aims at increasing energy efficiency by assessing the switching off possibilities of BSs, which are underloaded or have no load. The roaming-based network sharing method is adopted to further facilitate the switching off procedure allowing traffic offloading to BSs of all networks. In this section, we formulate the HetNet energy efficiency maximization problem and propose a heuristic solution to address it.

\section{A. Energy Efficiency Problem Formulation}

In order to increase network energy efficiency, denoted as $e e$, through roaming-based sharing and switching off, we consider the traffic load $J_{l}(t)$ of each BS $l$ for $t$ as the significant criterion for its operational state and the total HetNet's power consumption.

Let us express first the total bit rate demand of all HetNet UEs, $\rho^{\mathcal{L}}(t)$, in $A$ during hour $t . \rho^{\mathcal{L}(t)}$ is defined by the individual bit rate demands $\rho^{k}(t)$ of each $k \in \mathcal{K}(t)$, as well as by their association state $q_{k, l}(t)$ of $k$ with a $\mathrm{BS} l$ as

$$
\rho^{\mathcal{L}}(t)=\sum_{\forall k \in \mathcal{K}(t)} \sum_{\forall l \in \mathcal{L}} q_{k, l}(t) \cdot \rho_{k}(t) .
$$

Based on the above and in accordance with eq. (2), power consumption of total HetNet $\mathcal{L}$ is seen analytically in eq. (10). 


$$
P^{\mathcal{L}}(t) \quad=\quad \sum_{l=1}^{l=|\mathcal{L}|} \theta_{l}(t) \quad \cdot \quad\left(P_{l}^{c o n}+\Delta P_{l} \cdot \frac{P_{l}^{t x, \max }}{h_{l} \cdot J_{l, \max }} \cdot \sum_{k \in \mathcal{K}(t)} q_{k, l}(t) \cdot\left\lceil\frac{\rho_{k}(t)}{W_{l}^{R B} f\left(S N R_{k, l}(t)\right)}\right\rceil\right)
$$

Consequently, the network energy efficiency problem can be expressed in mathematical terms as follows

$$
\begin{array}{ll}
\max _{\theta_{l}, q_{k l}} & \operatorname{ee}\left(\theta_{1}(\mathrm{t}), \mathrm{q}_{\mathrm{k}, 1}(\mathrm{t})\right)=\frac{\rho^{\mathcal{L}}\left(\mathrm{q}_{\mathrm{k}, l}(\mathrm{t})\right)}{P^{\mathcal{L}}\left(\theta_{\mathrm{l}}(\mathrm{t}), \mathrm{q}_{\mathrm{k}, 1}(\mathrm{t})\right)} \\
\text { s.t. } & \sum_{\forall l \in \mathcal{L}} \theta_{l}(t) \leq|\mathcal{L}|, \theta_{l}(t) \in\{0,1\}, \\
& \sum_{\forall l \in \mathcal{L}} q_{k, l}(t) \leq 1, q_{k, l}(t) \in\{0,1\}, \\
& J_{l}(t) \leq J_{l, \text { max }}, \\
& \sum_{\forall l \in \mathcal{L}} \theta_{l}(t) \cdot J_{l}(t) \leq \sum_{\forall l \in \mathcal{L}} J_{l, \max } .
\end{array}
$$

Constraint (11b) reassures that only the BSs of the cooperative MNOs are studied and that a BS can interchange its operating state only between active and inactive. Constraint (11c) ensures that a UE $k$ can only be served by one BS $l$, eNB or $\mathrm{SC}$, and is considered non-associated otherwise. Moreover, the total traffic load $J_{l}(t)$ of an $l$ is limited by its own maximum capacity, $J_{l, \max }$ according to constraint (11d), while the total traffic load of the active BSs in the studied network cannot exceed the total maximum network capacity, as indicated by constraint (11e).

The problem of eq. (11) is an NP-hard, non-linear integer problem [29], [30]. This is due to the fact that the solution of problem in eq. (11) typically requires searching big search trees of possible configurations of BS operation states $\left(\theta_{l} \in\{0,1\}\right)$ and UE associations $\left(q_{k, l} \in\{0,1\}\right)$ that result in different energy efficiency values. In order to address the problem, while taking into consideration cooperation issues of MNOs, such as which BS of which MNO to deactivate, we propose a greedy heuristic scheme. Our proposal, Cooperative Switching Off Algorithm (CSO), constitutes a switching off scheme suitable for application to a multi-operator shared HetNet and is described in detail in the following Section IV-B.

\section{B. Cooperative Switching Off Algorithm (CSO)}

We propose a greedy heuristic algorithm which aims at reducing the energy consumption of a network by applying the method of switching off to both eNBs and SCs [31]. At the same time, it offloads the UEs of switched off BSs to neighbouring cells of the unified HetNet, as a result of roamingbased sharing among MNOs. The proposed scheme, namely Cooperative Switching Off (CSO), considers as switching-off criterion the traffic load of each $l \in \mathcal{L}$ in combination with an energy and roaming-cost related UE re-association scheme and is of centralised application. Being a greedy heuristic scheme, CSO follows the mentality of greedy heuristic schemes. In detail, given a starting solution, the greedy algorithm directs it to an updated solution that gives the largest increase (or reduction, depending on the problem) in the objective of the studied problem. This procedure is repeated until no increase in the objective can be obtained. CSO implements this philosophy following four steps: (i) Initial Setting for setting the starting solution, (ii) Execution of Greedy Component for executing the loop that finds the solution that achieves an energy efficiency increase, (iii) Acceptance criterion for updating the solutions with the ones that achieve larger increase and (iv) Repetition stage and Termination criterion for repeating the procedure until no increase in the objective, i.e., increase of network energy efficiency, can be obtained. CSO is executed during low-traffic hours (i.e., 00:00 - 07:00), on an hourly basis, and is depicted in Algorithm 1 and Fig. 2. Its steps are described as follows:

1) Initial Setting: Before the application of $\mathrm{CSO},(t=1)$, networks are considered to be full operational (FON), i.e., all BSs are in active mode and no cooperation scheme exists among MNOs. Then, BS operational states, UE association states of FON and thus, respective network BS traffic load and energy efficiency of FON are set as an input for the procedures of CSO that follow $\left(\theta_{l}^{I n}, q_{k, l}^{I n}, J_{l}^{I n}\right.$ and $e e_{\delta}^{I n}$, respectively, where $\delta$ represents an execution step of CSO). Otherwise, i.e., when $t \neq 1$, each of aforementioned values is set equal to the respective results of hour $(t-1)$.

2) Execution of Greedy Component: The greedy part of CSO constitutes the main body of the algorithm and is composed of a BS switching off assessment along with a UE re-association assessment. The BS switching off assessment is described in detail in the present section. The UE reassociation assessment is presented in the present section and described in detail in Section IV-C.

(i) BS Switching off assessment

It aims at saving energy by reducing the number of active BSs. Number of active BSs, their respective traffic load and UE association states are assessed virtually during this part in order to estimate their final values. Its basic steps consist of:

- Estimation of traffic demands: Expected number of UEs $\mathcal{K}(t)$ with their respective bit rate demands $\rho_{k}(t)$ for the following hour $t$ are estimated at the beginning of the hour. Thus, average data traffic demands are calculated in accordance to eq. (9).

- Choice of BS for application of the switching off method: The traffic load $J_{l}(t)$ of an $l$ during $t$ is calculated according to eq. (3) and constitutes the criterion for assessing switching off $l$ during $t$. MNOs are sorted in ascending order and according to their subscribed bit rate demands to initiate switching off assessing procedure. Then, they are studied consecutively. According to the scheme, the eNB of studied $n$ is assessed first to switch off, since eNBs are the most power consuming nodes of the network. Despite being the most power consuming node though, eNBs provide the highest coverage. Thus, through the whole procedure of $\mathrm{CSO}$, at least one of the collocated eNBs, $1 \leq l \leq|\mathcal{M}|$, remains active in $A$. 


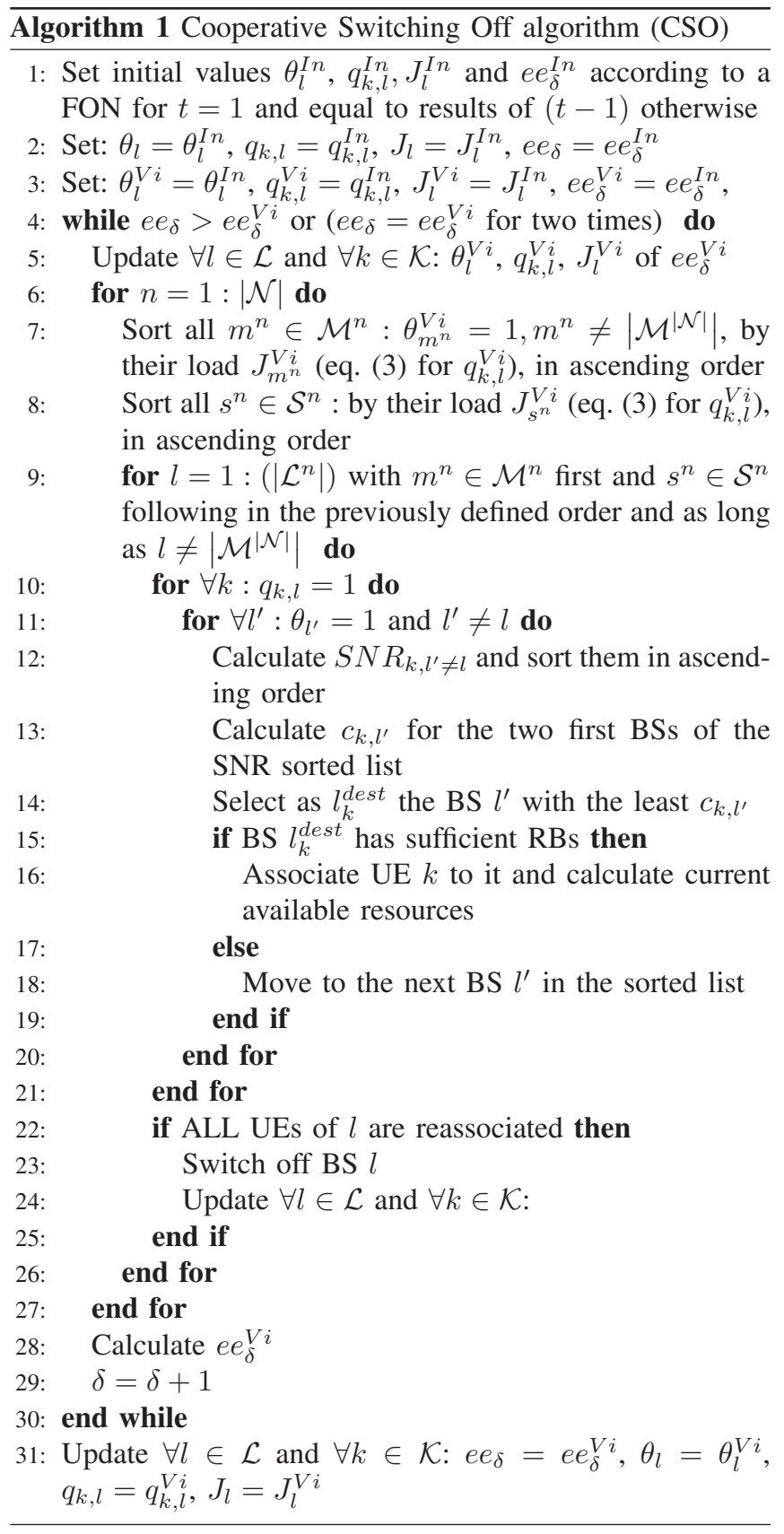

Since MNOs with the least bit rate demands are assessed first to enhance network switching off possibilities, an eNB of the MNO with the most traffic load, $l=|\mathcal{M}|$, remains active. CSO proceeds with assessing switching off possibilities of the rest BSs of studied $n$, i.e., its SCs, which are sorted by their $J_{l}(t)$ in ascending order. CSO starts from the least loaded one. Aiming at better coverage, SCs may transit between active and inactive operation state over the hourly application of CSO, depending on the expectations over traffic demands in $A$ during $t$. Unlike SCs and in order to avoid extensive extra power consumption, eNBs remain inactive during all studied hours, once it is decided by CSO as such.

- Application of UE re-association scheme: Associated to $l$ UEs need to be offloaded to other BSs so that $l$ can switch off. The scheme is based on $(i)$ the channel conditions between a UE $k$ and a destination $\mathrm{BS} l^{\prime}$ to which $k$ may get associated to $\left(S N R_{k, l^{\prime}}(t)\right)$, and (ii) the respective roaming charges. Roaming charges for $k, c_{k, l^{\prime}}$, are considered dependent on the type and owner MNO of $l^{\prime}$. The UE re-association scheme and the considered roaming charges are described in detail in Section IV-C.

- End of Greedy component: If and only if $\forall k \in \mathcal{K}(t)$ for which $q_{k, l}^{V i}(t)=1, l \in \mathcal{L}$ and $l \neq|\mathcal{M}|$, can be reassociated to a neighbouring $l^{\prime} \in \mathcal{L}, l^{\prime} \neq l$, BS $l$ is eligible to switch off. Otherwise, it remains active. Virtual variables $\theta_{l}^{V i}, q_{k, l}^{V i}, J_{l}^{V i}$ are updated $\forall l \in \mathcal{L}$ and $\forall k \in \mathcal{K}$.

3) Acceptance criterion: If $e e_{\delta} \geq e e_{\delta}^{V i}$, then $e e_{\delta}$ is set equal to $e e_{\delta}^{V i}$. Otherwise, it remains equal to $e e_{\delta}$. Depending on the chosen value of $e e_{\delta}$, values of $\theta_{l}, q_{k, l}$ are $J_{l}$ updated accordingly.

4) Repetition stage and Termination criterion: Energy efficiency improvement is achieved through the repeated application of the two previous steps. As long as $e e_{\delta}>e e_{\delta}^{V i}$ or $e e_{\delta}=e e_{\delta}^{V i}$ for two steps $\delta$, the virtual values are normally applied to $(i)$ the real ones of the next step $(\delta+1)$, i.e., $e e_{\delta+1}=e e_{\delta}^{V i}$, while values of $\theta_{l}, q_{k, l}$ are $J_{l}$ updated accordingly, and (ii) to the virtual ones of the next step $(\delta+1)$, i.e., $e e_{\delta+1}^{V i}=e e_{\delta}^{V i}$, while values of $\theta_{l}, q_{k, l}$ are $J_{l}$ updated accordingly.

\section{Cooperative UA Scheme}

As previously described, the re-association scheme assesses the channel between a UE $k$ and a neighbouring BS $l^{\prime}$, as well as the cost of the re-association process. Apparently, there is a high probability for a $k$ to associate to an eNB, $l^{\prime}, 1 \leq l^{\prime} \leq|\mathcal{M}|$, as its transmitted signal is stronger. Nevertheless, eNBs are at the same time more load-dependent on power consumption. Therefore, in order to achieve further energy saving, the re-association scheme adopts a comparison between two $l^{\prime}$. The comparison constitutes a form of biasing on cell selection towards SCs $\left(|\mathcal{M}| \leq l^{\prime} \leq|\mathcal{L}|\right)$, thanks to the calculation of the re-association cost we present in eq. (12). The re-association scheme of a UE $k$ from a BS $l$ to a BS $l^{\prime}$ includes the following steps:

- For every $k, k \in \mathcal{K}$ for which $q_{k, l}=1$ with a studied BS $l$ : the two BSs $l_{1}^{\prime}$ and $l_{2}^{\prime}$ with the best, $S N R_{k, l_{1}^{\prime}}$ and $S N R_{k, l_{2}^{\prime}}$ and consequently with the minimum required RBs, $j_{k, l_{1}^{\prime}}$ and $j_{k, l_{2}^{\prime}}$, are selected.

- The re-association process cost of a UE $k$, subscriber of MNO $n$, to a destination BS $l^{\prime}$, owned by a different MNO $n^{\prime}$, is calculated as

$$
c_{k, l^{\prime}}(t)= \begin{cases}c^{p} q_{k, l^{\prime}}(t) j_{k, l^{\prime}}(t) P_{l^{\prime}}^{R B} \Delta P_{l^{\prime}} t, & n=n^{\prime} \\ c^{p} q_{k, l^{\prime}}(t) j_{k, l^{\prime}}(t) P_{l^{\prime}}^{R B} \Delta P_{l^{\prime}} t+c^{n} \cdot \rho_{k}, & n \neq n^{\prime}\end{cases}
$$

where $c^{p}$ is the fixed power consumption charge $(€ / \mathrm{kWh})$ and $c^{n}, n \in \mathcal{N}$, is the inter-operator charge $(€ / M B)$ set among the co-operative MNOs for providing their services to a roamed UE. It has to be noted that it is possible that each MNO defines a different $c^{n}$ in order to host the traffic of rival MNOs. 


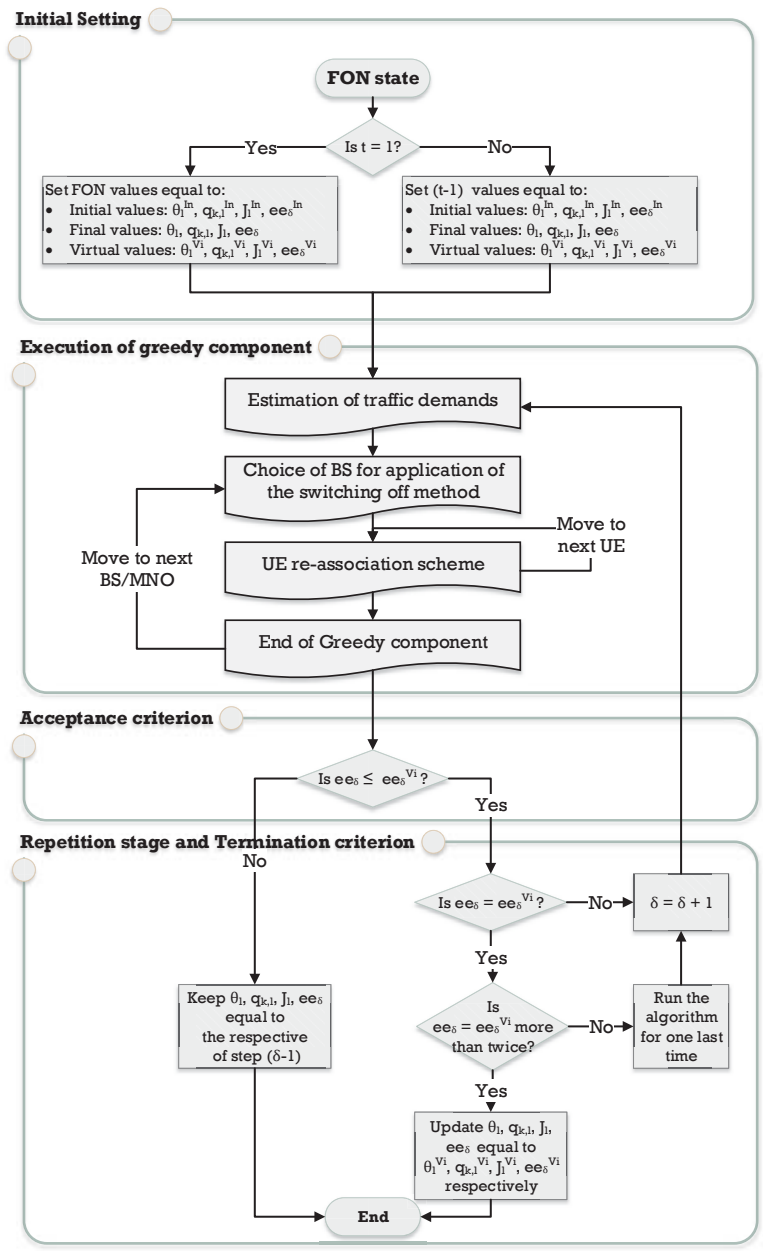

Figure 2. CSO flowchart.

- Finally, the UE $k$ is associated to the BS $l^{\prime}$ which results into the minimum re-association cost.

\section{CSO Complexity}

Based on the description of the CSO scheme in Sections IV-B and IV-C, multiple searches are implemented for the extraction of a solution from CSO. More specifically, an initial suboptimal configuration of BS operational states $\left(\theta_{l}\right)$ and UE associations $\left(q_{k, l}\right)$, along with their resulting BS traffic loads $\left(J_{l}\right)$ and network energy efficiency (ee), are provided as an input to CSO at the beginning of a studied period, $T=\left[t_{1}, t_{2}\right]$. Then, for each of the cooperative MNOs, a quick-sorting of all BSs $l \in \mathcal{L}$ based on their $J_{l}(t)$ is executed. Given that quick-sort complexity is $\mathcal{O}(n \cdot \log (n))$ and that no more than $|\mathcal{N}|=|\mathcal{L}|$ MNOs can be found in the studied system, the resulting complexity of CSO is $\mathcal{O}(|\mathcal{L}| \cdot|\mathcal{L}| \cdot \log (|\mathcal{L}|))$ or $\mathcal{O}\left(|\mathcal{L}|^{2} \cdot \log (|\mathcal{L}|)\right)$. However, the procedure of CSO continues with the assessment of each BS's switching off possibilities. In detail, for the BSs of each of the sorted HetNets $\left|\mathcal{L}^{n}\right|$, starting from the eNB and proceeding to SCs, the UE re-association scheme of Section IV-C is applied. Thus, for each associated UE $k$, a BS quick-sorting in executed based on the in-between them SNR $\left(S N R_{k, l}\right)$. After all the UEs of the studied to switch off $l$ have been considered, a BS quick-sorting may be necessary again so that all BS traffic loads, $J_{l}(t)$, are updated. Given the two quick-sorting procedures that take place and that the maximum number of UEs that can be associated to an $l$ is $\max (|\mathcal{K}(t)|)$, the complexity that is introduced by the procedure of the switching off assessment is

$\mathcal{O}(\max (|\mathcal{K}(t)|) \cdot|\mathcal{L}| \cdot \log (|\mathcal{L}|))+\mathcal{O}(|\mathcal{L}| \cdot \log (|\mathcal{L}|))=$ $\mathcal{O}(\max (|\mathcal{K}(t)|) \cdot|\mathcal{L}| \cdot \log (|\mathcal{L}|))$.

The total complexity of CSO then becomes:

$\mathcal{O}\left(|\mathcal{L}|^{2} \cdot \log (|\mathcal{L}|)\right) \cdot \mathcal{O}(\max (|\mathcal{K}(t)|) \cdot|\mathcal{L}| \cdot \log (|\mathcal{L}|))=$ $\mathcal{O}\left(\max (|\mathcal{K}(t)|) \cdot|\mathcal{L}|^{3} \cdot \log ^{2}(|\mathcal{L}|)\right)=\mathcal{O}\left(|\mathcal{L}|^{3} \cdot \log ^{2}(|\mathcal{L}|)\right)$. It is noted though that for realistic scenarios, CSO can provide results in acceptable executable time.

\section{Cost allocation Problem}

The proposed energy efficient solution is based on network sharing. Thus, MNOs need to be permanently motivated to refrain from an individual application of the switching off method and instead keep their cooperation holding. The latter presumes not only that cooperative MNOs reduce their expenses, but also that a fair cost allocation among them is adopted. A predefined and applied on an set basis agreement could achieve the desirable profitability and fairness. The cost that will be allocated to each MNO after its participation in a coalition agreement should mirror its contribution to the network cost savings acquired from the application of CSO algorithm. Moreover, revenues obtained from the roamed traffic served by rival host MNOs should not be neglected. The final cost allocation should be validated as fair by each MNO $n \in \mathcal{N}$, meanwhile being profitable in comparison to its noncooperative action.

\section{A. Cost allocation Problem Formulation}

Let $\Omega \subseteq \mathcal{N}$ be a coalition of MNOs who cooperate in order to apply the switching off algorithm CSO on their shared network. Each coalition $\Omega$ leads to an amount of total cost $E^{\mathcal{L}}\{\Omega\}$ due to energy consumption of formed HetNet, which all MNOs $n \in \Omega$ are responsible to share and pay. Given that we study the low-traffic hours, which correspond to a period $T=\left[t_{1}, t_{2}\right]$ and that $P^{\mathcal{L}^{n}}$ is calculated according to eq. (10), with $\mathcal{L}^{n}$ and $l^{n}$ instead of $\mathcal{L}$ and $l$, respectively, $E^{\mathcal{L}}\{\Omega\}$ is

$$
E^{\mathcal{L}}\{\Omega\}=c^{p} \sum_{n \in \Omega} \sum_{t \in T} P^{\mathcal{L}^{n}} t .
$$

If $\phi_{n}\{\Omega\}$ is the part of $E^{\mathcal{L}}\{\Omega\}$ that each $n \in \Omega$ will eventually take on to pay, we will proceed to its calculation by defining $(i)$ the roaming revenues corresponding to each $n$ when it participates in coalition $\Omega, \operatorname{rev}_{n}\{\Omega\}$, (ii) the contribution $\Phi_{n}^{\prime}\{\Omega\}$ of each $n$ to the total cost savings, $S E^{\mathcal{L}}\{\Omega\}$, between a cooperative and non-cooperative application of CSO and (ii) the expenses $\psi_{n}$ of $n$ when it individually (noncooperatively) applies CSO, as follows

$$
\phi_{n}\{\Omega\}=\psi_{n}-\Phi_{n}^{\prime}\{\Omega\}-\operatorname{rev}_{n}\{\Omega\} .
$$

Parameters $\psi_{n}$ and $\operatorname{rev}_{n}\{\Omega\}$ of $n \in \Omega$ can be easily calculated. More specifically, it is

$$
\psi_{n}=\sum_{t \in T} c^{p} P^{I n d, n}(t) t
$$


where $P^{I n d, n}(t)$ denotes the power consumption of $L^{n}$ in a non-cooperative application of $\mathrm{CSO}$, in accordance with eq. (10), with $\mathcal{L}^{n}$ and $l^{n}$ instead of $\mathcal{L}$ and $l$, and

$$
\begin{aligned}
& \operatorname{rev}_{n}\{\Omega\}= \\
& \sum_{t \in T} \sum_{k^{n} \in \mathcal{K}^{n}} \sum_{l^{n} \notin \mathcal{L}^{n}}\left(c^{p} q_{k^{n}, l^{n}}(t) j_{k^{n}, l^{n}}(t) P_{l^{n}}^{R B} \Delta P_{l^{n}} t+c^{n^{\prime}} p_{k^{n}}(t)\right)
\end{aligned}
$$

where $n^{\prime}$ is the MNO of coalition $\Omega$ to which UE $k$ is reassociated to and is other that provider $n \in \Omega$.

Unlike $\operatorname{rev}_{n}\{\Omega\}$ and $\psi_{n}$ of $n \in \Omega$ though, calculation of contribution $\Phi_{n}^{\prime}\{\Omega\}$ to energy cost savings $S E^{\mathcal{L}}\{\Omega\}$ implicates fairness issues, as other MNOs contribute to cost saving mainly by BS switching off and others mainly by serving offloaded traffic. It is possible that cooperative MNOs could proceed to an equal allocation of cost savings $S E^{\mathcal{L}}\{\Omega\}$ among them in order to determine $\Phi_{n}^{\prime}\{\Omega\}$ or proceed to an allocation based on their individual action. However, such strategies could result into a cost distribution that would possibly be unfair to other MNOs. Thus, we propose a more cooperative approach that could set aside possible fairness issues and provide an applicable solution to the described problem. To this end, we propose a centralised solution by modelling the energy allocation problem as a bankruptcy game.

A bankruptcy game combines the characteristics of a bankruptcy problem [20], [21] and cooperative games [22]. A bankruptcy game is defined by a specific entity that needs to be allocated among a group of players. Each of the players makes a claim on the obtained entity, while the sum of the players' claims cannot exceed it. A utility function is set for the game, according to cooperative game theory, which eventually allocates to each player a part of the entity, i.e., the payoff.

As a consequence, $S E^{\mathcal{L}}\{\Omega\}$ constitutes an entity that has to be completely allocated among the cooperative MNOs of a coalition $\Omega \subseteq \mathcal{N}$ so that $\Phi_{n}^{\prime}\{\Omega\}$ and eventually $\phi_{n}\{\Omega\}$ can be determined. Taking into consideration that different coalitions $\Omega$ can be formed among the MNOs, $S E^{\mathcal{L}}\{\Omega\}$ is calculated as follows

$$
S E^{\mathcal{L}}\{\Omega\}=\sum_{n \in \Omega} \psi_{n}-E^{\mathcal{L}}\{\Omega\} .
$$

It is noted that $\psi_{n}$ is calculated according to eq. (15) and serves as an upper limit of the eagerness of $n$ to participate in coalition $\Omega$. In other words, no MNO would be interested in paying an amount of cost greater than $\psi_{n}$ while, at the same time, no MNO could save more money than $\psi_{n}$ for a coalition in the case of a cooperation. Thus, $\psi_{n}$ represents the claim or "marginal cost", as we will name it hereafter.

The obtained allocation problem of the cost savings $S E^{\mathcal{L}}\{\Omega\}$ can be modelled as a bankruptcy problem, $B$, which allocates to each $n$ its contribution to the network cost savings, $\Phi_{n}^{\prime}\{\Omega\}$ and can be expressed in mathematical terms as

$$
B=\left\{\begin{array}{l}
\left(S E^{\mathcal{L}}\{\Omega\}, \psi_{n} \in \mathbb{R}_{++} \times \mathbb{R}_{+}^{|\Omega|}\right): \\
S E^{\mathcal{L}}\{\Omega\} \leq \sum_{n \in \Omega} \psi_{n},
\end{array}\right\}
$$

We use the coalitional game theory, and more specifically, the theory of coalitional games of transferable utility (TU), in order to define the characteristic function, $V_{B}, V_{B}: 2^{N} \times \mathbb{R}$, which will evaluate each bankruptcy problem $B$ and associate it to a real value:

$V_{B}(\Omega)=\left\{\begin{array}{lc}\min \left\{\psi_{n \in \Omega}, S E^{\mathcal{L}}\{\Omega\}-\sum_{n \notin \Omega} \psi_{n}\right\}, & \Omega=\{n\} \\ \min \left\{\sum_{n \in \Omega} V_{B}(\{n\}), S E^{\mathcal{L}}\{\Omega\}-\sum_{n \notin \Omega} \psi_{n}\right\}, \Omega \neq\{n\}\end{array}\right.$

It is noted that if $V_{B}(\Omega)<\left(S E^{\mathcal{L}}-\sum_{n \notin \Omega} \psi_{n}\right)$, then $V_{B}(\Omega)=0$, since the entity cannot be totally allocated to the cooperative MNOs.

Given the fact that $S E^{\mathcal{L}}\{\Omega\}$ is in any case defined only by the player MNOs who participate in the coalition, (19) becomes

$$
V_{B}(\Omega)=\left\{\begin{array}{lr}
\min \left\{\psi_{n \in \Omega}, S E^{\mathcal{L}}\{\Omega\}\right\}, & \Omega=\{n\} \\
\min \left\{\sum_{n \in \Omega} V_{B}(\{n\}), S E^{\mathcal{L}}\{\Omega\}\right\}, \Omega \neq\{n\},
\end{array}\right.
$$

with $V_{B}(\Omega)=0$ when $V_{B}(\Omega)<S E^{\mathcal{L}}$. Based on eq. (20), the value of the game among MNOs of coalition $\Omega, V_{B}(\Omega)$, is calculated based on the game values in case of an MNO individual activity, i.e., when $\Omega=\{n\}$.

Payoff of cost savings for each $n, \Phi_{n}^{\prime}\{\Omega\}$ or $\Phi_{n}^{\prime}\left(V_{B}(\Omega)\right)$, as it can be written in the case of the $B$ game with utility function $V_{B}$, is subjected to the following constraints so that the bankruptcy game holds:

- The sum of allocated payoffs should equal $V_{B}(\Omega)$ :

$$
\sum_{n \in \Omega} \Phi_{n}^{\prime}\left(V_{B}(\Omega)\right)=V_{B}(\Omega)
$$

- The payoff of a player $n$ in a coalition $\Omega$ should not be less than the respective one of the player's stand-alone action:

$$
\Phi_{n}^{\prime}(\{n\}) \leq \Phi_{n}^{\prime}\left(V_{B}(\Omega)\right) .
$$

- A player $n$ cannot receive a higher payoff than its claim, so that fairness is preserved.

$$
0 \leq \Phi_{n}^{\prime}\left(V_{B}(\Omega)\right) \leq \psi_{n} .
$$

\section{B. Bankruptcy game with Shapley Value}

We use Shapley Value (SV) to determine the payoffs, $\Phi_{n}^{\prime}\left(V_{B}(\Omega)\right)$, of each $n \in \Omega$, player of the bankruptcy game, with (20) set as the characteristic function which corresponds each game to a value. SV has the important characteristic of quantifying the contribution of a player, i.e., its worth and value, in a game when the player joins a coalition, which highly motivated us to selected it as part of our solution. Similarly, in the present work, SV rewards a player $n$ with a payoff $\Phi_{n}^{\prime}\left(V_{B}(\Omega)\right)$ according to its contribution in the obtained cost which. SV is based on four basic axioms [22]:

- Efficiency axiom: $\sum_{n \in \Omega} \Phi_{n}^{\prime}\left(V_{B}(\Omega)\right)=V_{B}(\Omega)$.

- Symmetry axiom: if two players $n_{1}$ and $n_{2}$ are such that $V_{B}\left(\Omega \cup\left\{n_{1}\right\}\right)=V_{B}\left(\Omega \cup\left\{n_{2}\right\}\right)$, for every coalition $\Omega$ containing the player $n_{1}$ and $n_{2}$, then $\Phi_{n_{1}}^{\prime}\left(V_{B}(\Omega)\right)=\Phi_{n_{2}}^{\prime}\left(V_{B}(\Omega)\right)$.

- Dummy axiom: If a player $n$ is such that $V_{B}(\Omega)=V_{B}(\Omega \cup\{n\})$, for every coalition $\Omega$ not containing $n$, then $\Phi_{n}^{\prime}\left(V_{B}(\Omega)\right)=0$.

- Additivity axiom: if $u$ and $v$ are characteristic functions, then $\Phi^{\prime}(u+v)=\Phi^{\prime}(v+u)=\Phi^{\prime}(u)+\Phi^{\prime}(v)$. 


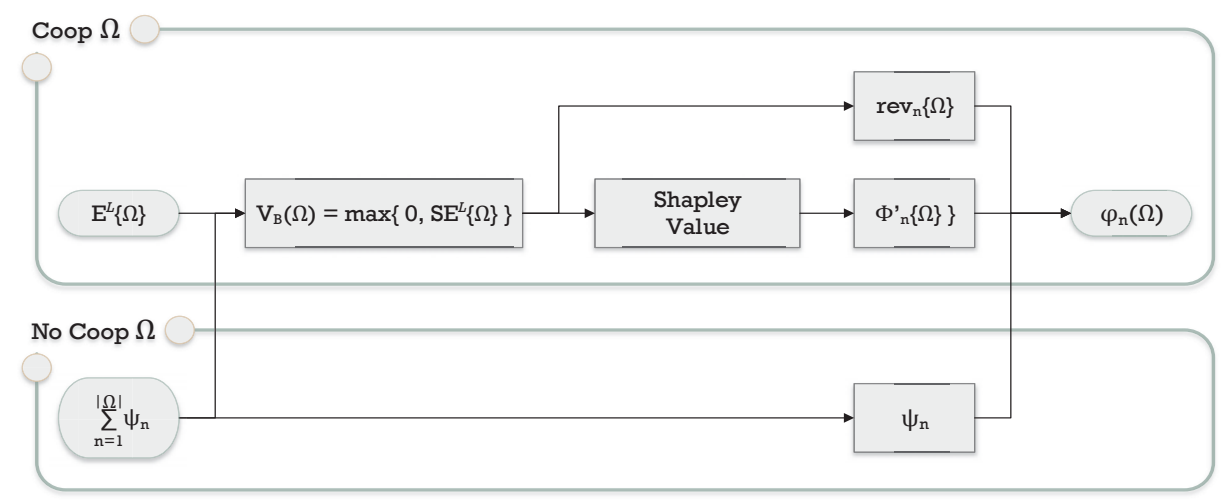

Figure 3. Flowchart of the bankruptcy Shapley Value based cost allocation scheme (BSV).

SV payoff of a player in a game when it joins coalition $\Omega$, is computed based on the chosen utility function of the game, $V_{B}$, as follows [22]

$$
\Phi_{n}^{\prime}\left(V_{B}(\Omega)\right)=\sum_{\Omega \subseteq \mathcal{N} \backslash\{n\}} \frac{|\Omega| !(N-|\Omega|-1) !}{N !}\left[V_{B}(\Omega \cup\{n\})-V_{B}(\Omega)\right] .
$$

With the contribution $\Phi_{n}^{\prime}\left(V_{B}(\Omega)\right)$ defined from SV, the cost $\phi_{n}\{\Omega\}$ that each $n$ will eventually take on to pay can be calculated according to eq. (14). Our proposal, namely bankruptcy Shapley Value based cost allocation scheme (BSV), is presented in Fig. 3.

Aiming at further portraying the fairness of the allocated cost to each MNO, $\phi_{n}\{\Omega\}$, we employ an expectation index, $E I_{n}\{\Omega\}$, corresponding to each $n \in \Omega$ defined as

$$
E I_{n}\{\Omega\}=\frac{\left(\psi_{n}-\phi_{n}\{\Omega\}\right)}{\psi_{n}}, \forall n \in \Omega
$$

where $\phi_{n}\{\Omega\}$ and $\psi_{n}$ are calculated according to eq. (14) and (15), respectively. The meaning of $E I_{n}\{\Omega\}$ is to portray a metric of satisfaction for an $n$ between the case of being in a coalition $\Omega$ and opting for an individual action, based on the achieved cost difference. An estimation for the whole method's fairness is extracted based on the mean value of $E I_{n}\{\Omega\}$.

\section{PERformance Evaluation}

We implemented a system-level simulator in MATLAB to examine the performance of the proposed CSO algorithm and bankruptcy cost allocation scheme. In the present section, the considered simulation scenario and the respective performance evaluation results are presented.

\section{A. Simulation Scenario}

We study seven macrocell sized areas $A$, with correspondence to the system model described in Section III. Without loss of generality, we focus on the case when $|\mathcal{N}|=3$ MNOs are active in each $A$. We consider that all MNOs are motivated to cooperate, aiming at maximizing the energy savings of their shared network and then proceed in balancing their expenses in a fair way. We consider uniform UE distribution in each $A$, with all MNOs having similar traffic load patterns of sinusoidal form, approaching the traffic pattern in [8], unless otherwise stated. Their traffic loads, expressed in number of UEs, are equal to $\xi \cdot\left|\mathcal{K}^{1}\right|,\left|\mathcal{K}^{2}\right|=2 \cdot \xi \cdot\left|\mathcal{K}^{1}\right|$ and $\left|\mathcal{K}^{3}\right|=3 \cdot \xi \cdot\left|\mathcal{K}^{1}\right|$. Parameter $\xi$ is a multiplicative real-number factor, $\xi \in \Re$, that is multiplied by the whole MNO traffic pattern curve, differentiating the load magnitudes for each MNO. Unless otherwise stated, $\xi$ is set equal to 1 . The network infrastructure in each $A$ is in total $|\mathcal{L}|=45$ with $\left|\mathcal{L}^{1}\right|=11,\left|\mathcal{L}^{2}\right|=15$ and $\left|\mathcal{L}^{3}\right|=19$. One eNB corresponds to each of the three MNOs from the aforementioned infrastructure. The eNBs are collocated in the centre of each $A$, while the rest, i.e., the $\mathrm{SCs}$, are uniformly distributed in it. We assume two possible operational states for the BSs, active and inactive and represent their state with the $\theta_{l}$ value, as mentioned in Section III-B. All MNOs adopt orthogonal LTE-A transmission of bandwidth $W_{l}=20 \mathrm{MHz}$. Therefore $J_{l, \max }=100 \mathrm{RBs}, \forall l \in \mathcal{L}$ [28]. We set $|\mathcal{I}|=3$ classes of data throughput demands. Thus, a UE $k$ may require $\rho_{1}=1.5 \mathrm{Mbps}, \rho_{2}=1 \mathrm{Mbps}$ or $\rho_{3}=0.75 \mathrm{Mbps}$ with random probability $g_{k, i} \in[0,1]$. We set $c^{p}=0.1 € / \mathrm{kWh}$ as a cost corresponding to power consumption while the interoperator roaming charge is set equal to $c^{n}=0.003 € / \mathrm{MB}$ [32]. Each $k, k \in \mathcal{K}$ is initially associated to an $l, l \in \mathcal{L}^{n}$ that is owned by the MNO $n$ to which $k$ is subscribed and that provides the highest $S N R_{k, l}$. We set the threshold SNR for the establishment of a channel $S N R_{\text {threshold }}=-10 \mathrm{~dB}$ [28]. The present study focuses on the low traffic hours of a day $(00: 00)-(07: 00)$, when switching off probabilities are higher. The rest of the simulation parameters are summarized in Table I [8], [27], [28], [33].

The proposed CSO algorithm is compared with (i) a full operational network (FON), when all $l \in \mathcal{L}$ are in active mode and no cooperation scheme exists among MNOs, (ii) an Individual network operation according to the proposed CSO (IndSO), when each MNO $n \in \mathcal{N}$ individually applies the switching off scheme of CSO, (iii) a random switching off scheme (RSO), when MNOs apply both the switching off and roaming-based network sharing method by switching off half BSs of the united network in a random manner so that the geographic area corresponding to a switched off $\mathrm{BS}, l^{\prime} \in \mathcal{L}$ is covered by the remaining active ones [23] and (iv) a switching off scheme that is based on a power saving efficiency indicator $Q$ and to which we will hereafter refer to as Q-base switching off scheme (QSO) [12]. As QSO is designed for a single- 
Table I

Simulation PARAmeter VAlues

\begin{tabular}{|c|c|}
\hline Parameter & Value \\
\hline \hline$h_{l \leq|M|}$ & 6 \\
\hline$h_{l>|M|}$ & 2 \\
\hline$P_{l \leq|M|}^{t x, m a x}$ & $46 \mathrm{dBm}$ \\
\hline$P_{l>|M|}^{t x, m a x}$ & $30 \mathrm{dBm}$ \\
\hline$G_{k, l \leq|M|}^{t x}$ & $15 \mathrm{dBi}$ \\
\hline$G_{k, l>|M|}^{t x}$ & $5 \mathrm{dBi}$ \\
\hline$P L_{k, l \leq|M|}$ & $128.1+37.6 \log d_{k, l}, d$ in km \\
\hline$P L_{k, l>|M|}$ & $140.7+36.7 \log d_{k, l}, d$ in $\mathrm{km}$ \\
\hline$N_{t h}$ & $-174 \mathrm{dBm} / \mathrm{Hz}$ \\
\hline$N F$ & $5 \mathrm{~dB}$ \\
\hline$\Delta P_{l \leq|M|}$ & 21.45 \\
\hline$\Delta P_{l>|M|}$ & 7.4 \\
\hline$P_{l \leq|M|}^{c o n}$ & $354.44 \mathrm{~W}$ \\
\hline$P_{l>|M|}^{c o n}$ & $71 \mathrm{~W}$ \\
\hline
\end{tabular}

operator system model, we adjust it in our results for a multioperator one by using a roaming-based shared network with traffic offloading to an eNB with available resources and giving priority to the eNB of their provider MNO. We also apply QSO on an hourly basis, as in our work.

The proposed BSV cost allocation scheme is compared with (i) an equal cost allocation (EQ) among the cooperative MNOs participants of a coalition $\Omega$, when each $n$ takes on the responsibility of paying an equal part of the network expenses except from the roaming revenues and (ii) the Generalized Ibn Ezra (GIE) Value [21], when the allocated cost $\phi_{n}^{G I E}\left(E^{H e t}, \psi_{n}\right)$ to each MNO $n$ is dependent on their marginal costs, $\psi_{n}$. Moreover, when necessary, we consider in our results the case when no cost allocation strategy is adopted (NoCA).

Finally, network energy efficiency (bits/Joule) and the corresponding network operation cost $(€)$ were used as indicative metrics for the validation of the algorithms performance results. Concerning the MNO satisfaction from the cost allocation methods, we use as a metric the EI which is given in (25), while the mean value of the EI is used for assessing the overall fairness of the methods.

\section{B. Performance Results}

Fig. 4 depicts the average network energy efficiency for the studied area throughout the low-traffic hours with the consideration of four different switching off schemes that were described in Section VI-A: IndSO, RSO, QSO and CSO. It can be observed that CSO outperforms its counterparts throughout the studied hours, since it manages to serve the same amount of traffic with less active infrastructure. QSO, performs worse than CSO in spite of the network sharing assumption, as it offloads traffic only to eNBs and does not exploit the available resources of other SCs, as CSO does. QSO though outperforms IndSO and RSO. On one hand, IndSO is outperformed by both CSO and QSO, because it does not apply network sharing. Moreover, in an $A$, IndSO keeps the eNBs of each MNO active and adjusts the operational states of SCs to traffic variations. In comparison to QSO, this results in higher power consumption for IndSO and thus, in lower network energy efficiency. On the other hand, RSO limits the amount of active infrastructure to half to save energy and provide coverage. In each $A$ thus, more than one eNB may

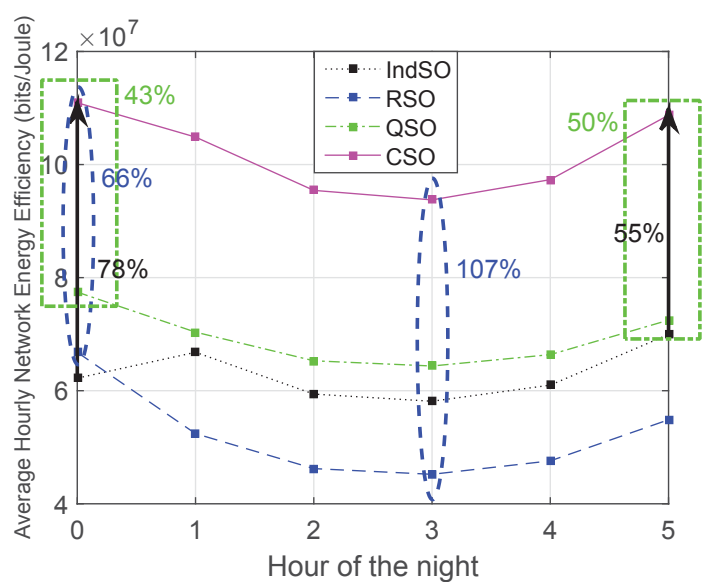

Figure 4. Average hourly network energy efficiency vs. switching off schemes.

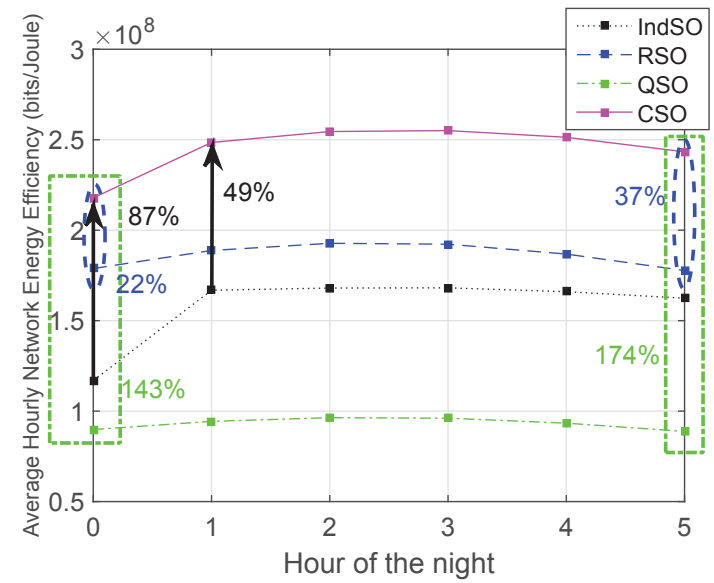

Figure 5. Average hourly network energy efficiency vs. switching off schemes with night peak MNO traffic profiles.

remain active, along with some underloaded SCs, which results in a poorer performance in comparison to its counterparts. Interesting is the fact that when traffic is a bit higher, i.e., 00:00-01:00, IndSO is outperformed by RSO, as the latter exploits more efficiently the active infrastructure. On the other hand, IndSO performs better than RSO for the rest of studied hours, since IndSO adjusts to traffic variations with SCs. In order to quantify the differences to its counterparts, it can be noticed that CSO can achieve an increase in energy efficiency between $55-78 \%$ in comparison to IndSO, between 66-107\% in comparison to RSO and $43-50 \%$ in comparison to QSO.

In order to study the performance of CSO under different traffic scenarios, we have also assumed a scenario where the load peaks of all MNOs take place during the night hours (e.g., at a student residence) in Fig. 5. A general comment for Fig. 5 is that all studied schemes are more energy efficient in comparison to their respective performances in Fig. 4, because higher traffic load is served by the networks. Among all schemes, CSO is the most energy efficient one, as it serves the traffic with less power consumption. In detail, it is mainly the network of MNO 3, that serves the majority of traffic. Its infrastructure is quite loaded, while some SCs of the other MNOs remain active as well. The MNO cooperation though allows eNBs and several SCs to switch off, which leads to energy savings. Although CSO performs better than all its 


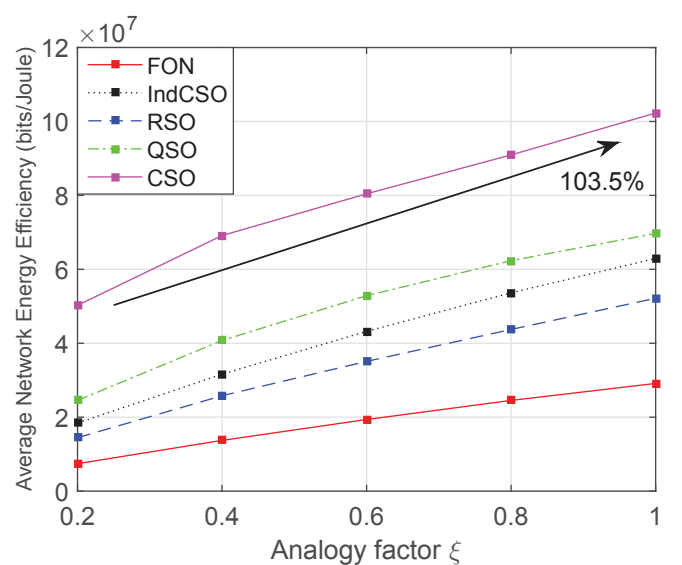

Figure 6. Average network energy efficiency vs. analogy factor $\xi$ and switching off schemes.

counterparts in Fig. 5 as in Fig. 4, the performance results of the rest considered schemes have been altered. RSO has now the second best performance. It is outperformed only by $\mathrm{CSO}$, because it keeps more eNBs active in each $A$. However, its energy efficiency results are better than those of QSO and IndSO. With RSO, more traffic is served by SCs, whereas with QSO and IndSO, all eNBS are active and take on the majority of traffic. QSO is outperformed by IndSO in the present figure despite the adoption of network sharing, because IndSO applies offloading to both eNBs and SCs, whereas QSO allows $\mathrm{SC}$ traffic offloading only to eNBs. Concluding, in Fig. 5, CSO achieves an increase in energy efficiency between $49-87 \%$ in comparison to IndSO, between $22-37 \%$ in comparison to RSO and $143-174 \%$ in comparison to QSO.

In an effort to obtain further insights on the performance of the studied schemes in relevance to energy efficiency, we assume different traffic load analogies among the cooperating MNOs, for the normal sinusoidal traffic pattern. The respective results are apparent in Fig. 6. Having already assumed MNOs of different traffic load, as described in Section VI-A, a cooperation among MNOs whose traffic load discrepancies change could result in a different load offloading among BSs and thus alter the energy efficiency of the considered system for the different switching off schemes. According to Fig. 6, CSO outperforms its counterparts for low and high traffic load differences, as they change by $\xi$, thanks to the joint consideration of its switching off and infrastructure sharing scheme. At the same time though, CSO improves its energy efficiency by $103.5 \%$ among the different cases of traffic load analogy, while the respective numbers for its counterparts are $184.2 \%$ for QSO, $260.9 \%$ for RSO, $241.8 \%$ for IndSO and $292.7 \%$ for FON. The more moderate responses to traffic load changes of CSO, QSO and IndSO in comparison to RSO and FON are attributed to the fact that the former increase the percentage of active infrastructure to cover the traffic, whereas the latter maintain it stable.

The content of Fig. 6 is extended in Fig. 7 out of interest to assess the effects of the different coalitions that MNOs can form on the performance of CSO, in terms of energy efficiency. In more detail, three kinds of coalition can be noticed: (i) one MNO (blue dashed line), (ii) two MNOs (black dotted

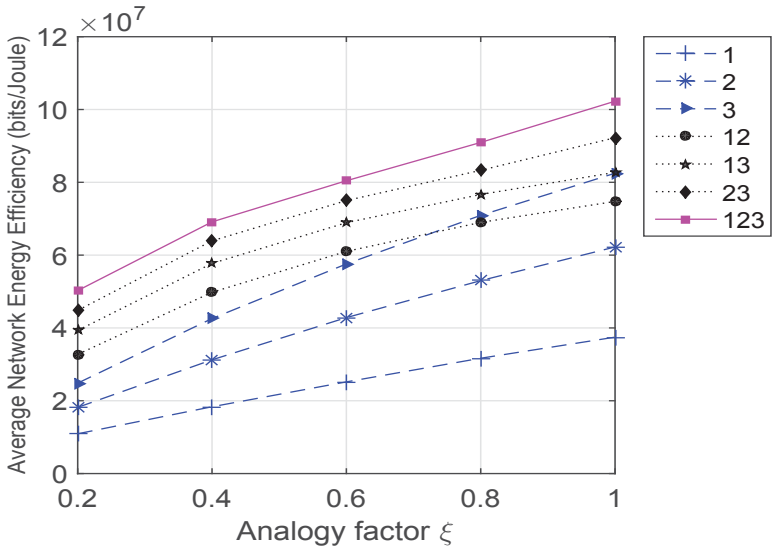

Figure 7. Average Network Energy Efficiency vs. analogy factor $\xi$ and Coalitions.

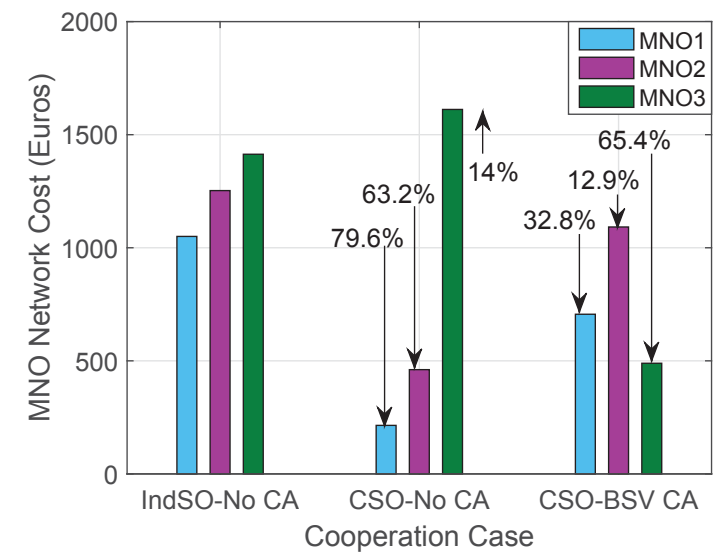

Figure 8. Average MNO network cost including roaming revenues vs. cooperation case and cost allocation strategy.

line) and (iii) three MNOs (pink solid line) applying the CSO scheme. It has to be noted that a coalition with one MNO (case $(i))$ represents the application of the IndSO scheme, as it was described in Section VI-A. According to the figure, in most cases, IndSO proves to be less energy efficient in comparison to any other coalition MNOs can form, since it does not allow MNOs to reap the benefits of sharing, i.e., offloading their traffic to any BS of the area. Comparing a coalition of two and three MNOs, the coalition of three proves to be more energy efficient that a coalition of two. Even though the amount of traffic that needs to be offloaded increases with the consideration of more cooperative MNOs, the traffic can be better distributed among the extra infrastructure that is available from the extra participant MNO, increasing thus the network energy efficiency.

Fig. 8 is an introduction to the distribution of costs to each of three considered MNOs in the case $(i)$ when all MNOs act individually and thus no cost allocation method is necessary (IndSO-NoCA). MNOs, however, are responsible for the expenses of their own network. Case (ii) corresponds to a coalition of the three MNOs, with no cost allocation method being adopted (CSO-NoCA). Instead, MNOs are responsible for the cost corresponding to their active infrastructure and roaming revenues. Finally, case (iii) represents a union of MNOs in a coalition of three and the adoption of the proposed 


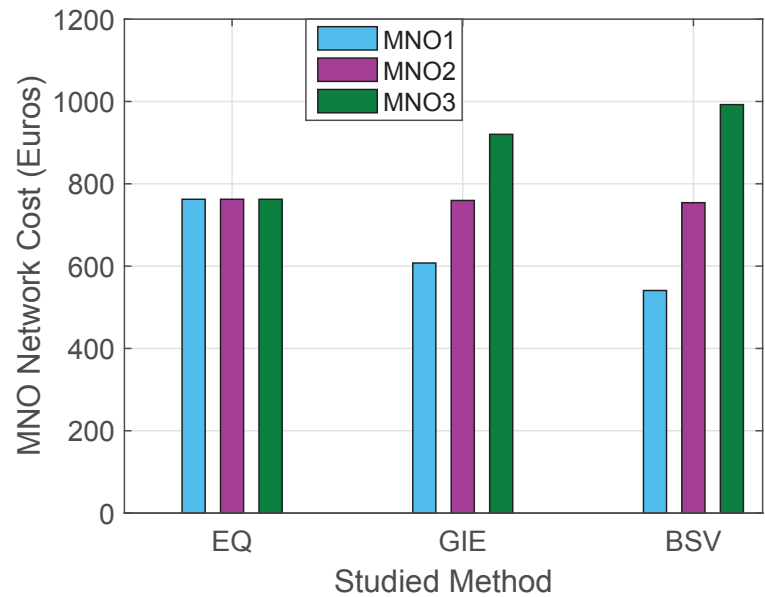

(a) Average MNO network cost vs. cost allocation methods.

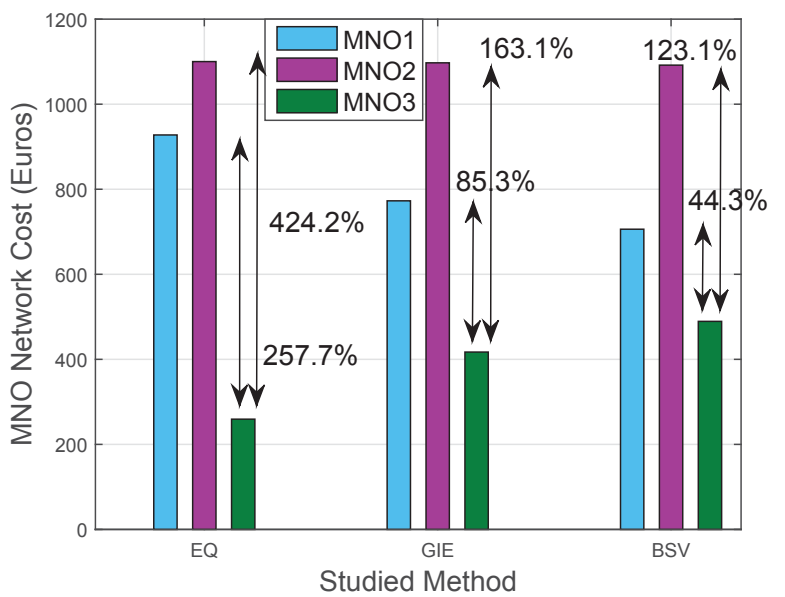

(b) Average MNO network cost including roaming revenues vs. cost allocation methods.

Figure 9. Allocation of the cost to cooperative MNOs considering different cost allocation methods before and after the consideration of inter-operator roaming revenues.

BSV cost allocation method (CSO-BSV). As can be seen, in the CSO-NoCA case, there is a big decrease of $79.6 \%$ and $63.2 \%$ for the network costs of MNOs 1 and 2, respectively, in comparison to the IndSO-NoCA case, as they are the MNOs whose infrastructure is assessed first to switch off, according to the CSO algorithm. Despite the fact that the benefits of infrastructure sharing are already apparent for MNOs 1 and 2, MNO 3 notices an increase of $14 \%$ in its costs, since it takes on the majority of the traffic. With the need for applying a fair cost allocation procedure among the cooperative MNOs being highlighted, the respective results for the CSO-BSV case are included in Fig. 8 as well. BSV cost allocation indicates as well a decrease of $32.8 \%$ and $12.9 \%$ in the costs of MNOs 1 and 2 respectively, in comparison to their respective marginal cost, i.e., IndSO-NoCA case. Both cost reductions, however, are more limited in comparison to the CSO-NoCA case, as BSV allows MNOs 1 and 2 to take on a larger share of the total costs. As far as MNO 3 is concerned, a big decrease of $65.4 \%$ is noticed in its allocated cost between the IndSO-NoCA and CSO-BSV cases, in contrast to the cost increase noticed in the CSO-NoCA one. The BSV cost allocation method rewards the contribution of MNO 3 to provide service to the offloaded traffic for limiting its switching off possibilities. MNO 3 thus is ultimately motivated to remain in the coalition. The consideration of Shapley Value, which is used in BSV, contributes so that all MNOs reduce their expenses and remain encouraged to share their networks by adopting a cost allocation that portrays each one's contribution to the cost savings.

Fig. 9 presents the cost distribution among the MNOs who participate in a coalition $\Omega$, as a result of different cost allocation methods. On one hand, Fig. 9(a) displays the influence of the considered methods on the cost that is attributed to the network power consumption. EQ cost allocation does not take into consideration any individual contribution of each MNO and thus the allocated costs are equal. The GIE method, being based on the declared marginal cost of each MNO, results in a more balanced cost allocation vector, especially between MNOs 1 and 3. Lastly, given that the BSV method portrays the contribution of each MNO to the cost savings, it approaches the GIE method. On the other hand, Fig. 9(b) depicts the results of Fig. 9(a), as they are formed after the consideration of each MNO's respective roaming revenues, $r e v_{n}$. The results of Fig. 9(b) constitute the final allocated costs. When roaming charges are considered after an EQ cost allocation, big discrepancies appear between MNO 3 and MNOs 1, 2. According to the figure, MNOs 1 and 2 approach their marginal costs and MNO 3 considerably benefits from the roaming charges. Undoubtedly, the big differences of $257.7 \%$ and $424.2 \%$ between MNO 3 and MNOs 1 and 2, respectively in their allocated costs, raise questions on the fairness of the method. The GIE and BSV methods however, manage to reduce the big gap between MNO 3 and MNOs 1 and 2, thanks to their criteria. BSV achieves a more balanced cost allocation between MNO 3 and MNOs 1 and 2, with respective differences of $44.3 \%$ and $123.1 \%$, in comparison to GIE, where the respective differences are equal to $85.3 \%$ and $163.1 \%$. It has to be noted that, for the three methods, MNO 2 has the least decrease in its allocated cost due to its large roamed traffic load, which induces larger roaming expenses.

The satisfaction of MNOs from the distribution of cost among them, as it was extracted from the different cost allocation methods (Fig. 9(b)), is expressed in Fig. 10, via the EI metric of (25). Moreover, the CSO-NoCA case of Fig. 8 is also included in the results. According to the figure, MNO 3 has a negative EI in the NoCA case, as the fact that it takes on the majority of the traffic results in increased expenses. It is noticed that when a cost allocation strategy is applied, EI of MNO 3 increases, indicating its satisfaction over the fact that it received a cost reduction as a payoff for providing a considerable part of its network capacity to cover the traffic of MNOs 1 and 2. On the other hand, MNOs 1 and 2, are more satisfied in the NoCA case, i.e., they have higher EI, since they have to pay only for the power consumption of their limited active infrastructure. When a cost allocation method is 


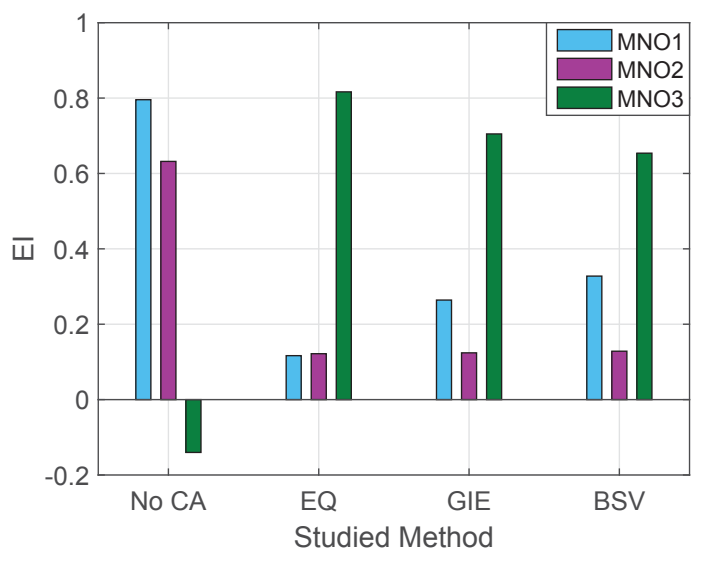

Figure 10. MNO EI vs. cost allocation methods.

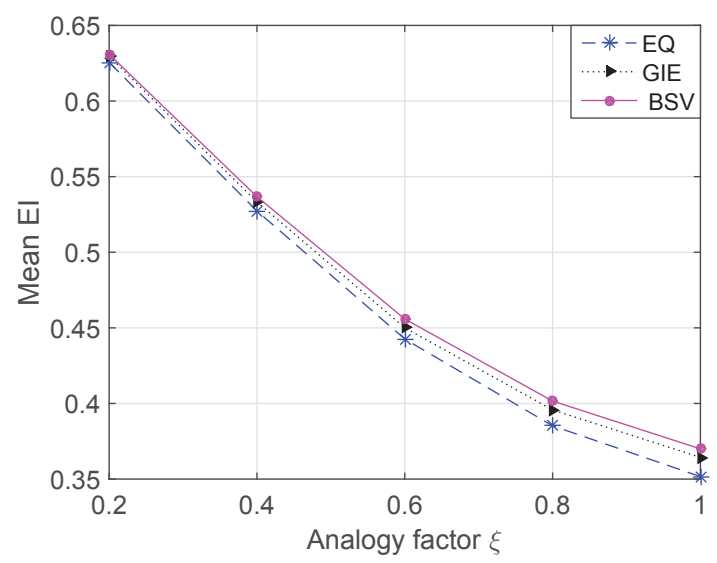

Figure 11. Mean EI of each cost allocation method vs. analogy factor $\xi$ for the MNO traffic loads.

applied, MNOs 1 and 2 have to take on a larger part of the total network expenses, limiting their individual satisfaction from the cooperation. However, an adoption of a cost allocation method leads to a more balanced distribution of the cost and consequently the reassurance of a minimal satisfaction of all cooperative MNOs. Among the cost allocation methods, the EQ one results in the most unbalanced MNO satisfaction, favouring MNO 3. However, GIE and BSV balance the EQ's method differences of EI for the cooperative MNOs thanks to the adoption of cost allocation criteria (marginal cost and saved energy contribution respectively), with the BSV criteria achieving the most balanced EI for the cooperative MNOs.

Having considered the results of Fig. 10, it is only rational to assume that the BSV balanced cost allocation among all the cooperative MNOs, which is also representative of their contribution to the achieved reduction of the cost, is a more fair option to follow. Fig. 11 confirms the latter conclusion with the display of the mean EI of each cost allocation method, $\mathrm{EQ}, \mathrm{GIE}$ and BSV, versus the analogy factor $\xi$ of the MNO traffic loads. According to the obtained results, all studied methods provide a fairness of similar levels in the system for various traffic load differences among the MNOs. Despite the fact that all studied cost allocation methods result in close performance, especially when traffic load differences are vague among the MNOs, the performance gap among the methods slightly expands as traffic load differences intensify as well. The BSV method though presents a slight precedence that is attributed to the use of Shapley Value, as the latter manages to quantify the contribution of each MNO to cost savings for the final extraction of their allocated costs. The GIE method, being based to the criterion of the marginal cost of each MNO performs close, though still below, to BSV for the various traffic load abnormalities. Finally, there is a slightly more intense deterioration in the performance of the EQ method in comparison to the others as the differences in the traffic load volume of each MNO broaden, which indicates the necessity of adopting fairer cost allocation criteria.

\section{CONCLUSION}

With a view to the expected increase in the traffic load volume during the following years, the possibility for MNOs to switch off infrastructure along with a possible cooperation among them expand the expectations for a greener operation of the telecommunication infrastructure that will reduce OpEx and $\mathrm{CO}_{2}$ emissions and increase energy savings. At the same time though, fairness issues over the cost allocation among the cooperative MNOs which could jeopardise their willingness for a cooperation are raised. In this paper, we introduced a switching off algorithm, CSO, which can achieve an energy efficient operation of the network during the low traffic hours, when the infrastructure is underloaded and exploit the benefits of network sharing by roaming traffic to the networks of all cooperative MNOs. By taking into consideration the different types of BSs composing the HetNet and their owner MNO, as well as the channel quality among BSs and UEs in the studied area, the proposed scheme assesses the best candidate BS to switch off, implements a roaming cost based cooperative UA scheme to offload traffic and eventually defines the operational state of the BSs in the area. The obtained results highlight not only the potential energy efficiency gains of the network, but also the potential cost savings. In order to address the fairness issues over the cost allocation among MNOs who cooperate, we proposed a bankruptcy game as a cost allocation method, BSV, which is Shapley Value based and thus takes into account each MNO's contribution to network cost savings according to their power consumption cost in a cooperative and non-cooperative case. The proposed BSV cost allocation scheme eventually determines the final cost to be allocated to each MNO based on their corresponding roaming revenues. According to the extracted results, the adaptability of the proposed scheme to provide a balanced cost allocation among MNOs for their different traffic loads while managing to be overall satisfying as well, is projected.

\section{REFERENCES}

[1] "Cisco visual networking index: Global mobile data traffic forecast update, 2016-2021," Cisco, Feb. 2017.

[2] E. Oh, B. Krishnamachari, X. Liu, and Z. Niu, "Toward dynamic energyefficient operation of cellular network infrastructure," IEEE Communications Magazine, vol. 49, no. 6, pp. 56-61, Jun. 2011.

[3] "Infrastructure sharing among MNOs," Applied Value Telecom Series, 2014.

[4] 3rd Generation Partnership Project, "Network sharing; Architecture and functional description," 3GPP TR 23.251, vol. 13.1.0, Rel. 13, Mar. 2015. 
[5] "CMS network sharing study 2016; Sharing gathers pace," CMS Legal Services EEIG, Apr. 2016.

[6] A. Khan, W. Kellerer, K. Kozu, and M. Yabusaki, "Network sharing in the next mobile network: TCO reduction, management flexibility, and operational independence," IEEE Communications Magazine, vol. 49, no. 10, pp. 134-142, Oct. 2011.

[7] "Market drivers for multi-operator small cells," Small Cell Forum, Jan. 2016.

[8] G. Auer, V. Giannini, C. Desset, I. Godor, P. Skillermark, M. Olsson, M. A. Imran, D. Sabella, M. J. Gonzalez, O. Blume, and A. Fehske, "How much energy is needed to run a wireless network?" IEEE Wireless Communications, vol. 18, no. 5, pp. 40-49, Oct. 2011

[9] "Base Station Planning Permission in Europe," GSMA TM., Dec. 2013. [Online]. Available: http://www.gsma.com/publicpolicy/basestation-planning-permission-in-europe

[10] J. Kim, W. S. Jeon, and D. G. Jeong, "Base-station sleep management in open-access femtocell networks," IEEE Transactions on Vehicular Technology, vol. 65, no. 5, pp. 3786-3791, May 2016.

[11] J. Zheng, Y. Cai, X. Chen, R. Li, and H. Zhang, "Optimal base station sleeping in green cellular networks: A distributed cooperative framework based on game theory," IEEE Transactions on Wireless Communications, vol. 14, no. 8, pp. 4391-4406, Aug. 2015.

[12] S. Cai, Y. Che, L. Duan, J. Wang, S. Zhou, and R. Zhang, "Green 5G heterogeneous networks through dynamic small-cell operation," IEEE Journal on Selected Areas in Communications, vol. 34, no. 5, pp. 1103 1115, May 2016

[13] C. Liu, B. Natarajan, and H. Xia, "Small cell base station sleep strategies for energy efficiency," IEEE Transactions on Vehicular Technology, vol. 65, no. 3, pp. 1652-1661, Mar. 2016.

[14] A. Bousia, E. Kartsakli, A. Antonopoulos, L. Alonso, and C. Verikoukis, "Multiobjective auction-based switching-off scheme in heterogeneous networks: To bid or not to bid?" IEEE Transactions on Vehicular Technology, vol. 65, no. 11, pp. 9168-9180, Nov. 2016.

[15] _ _ "Sharing the small cells for energy efficient networking: How much does it cost?" in 2014 IEEE Global Communications Conference, Dec. 2014, pp. 2649-2654

[16] L. Cano, A. Capone, G. Carello, and M. Cesana, "Evaluating the performance of infrastructure sharing in mobile radio networks," in 2015 IEEE International Conference on Communications (ICC), Jun. 2015 , pp. 3222-3227.

[17] Y. Bao, J. Wu, S. Zhou, and Z. Niu, "Bayesian mechanism based inter-operator base station sharing for energy saving," in 2015 IEEE International Conference on Communications (ICC), Jun. 2015, pp. 4954.

[18] B. Leng, P. Mansourifard, and B. Krishnamachari, "Microeconomic analysis of base-station sharing in green cellular networks," in IEEE INFOCOM 2014 - IEEE Conference on Computer Communications, Apr. 2014, pp. 1132-1140.

[19] M. Gruber and S. Borst, "Fair decisions in shared multi-operator mobile networks," in 2015 IEEE 26th Annual International Symposium on Personal, Indoor, and Mobile Radio Communications (PIMRC), Aug. 2015, pp. 1493-1497.

[20] M. Pulido, J. Sánchez-Soriano, and N. Llorca, "Game theory techniques for university management: An extended bankruptcy model," Annal of Operations Research, vol. 109, no. 1, pp. 129-142, 2002. [Online]. Available: http://dx.doi.org/10.1023/A:1016395917734

[21] J. Alcalde, M. d. C. Marco, and J. A. Silva, "Bankruptcy games and the Ibn Ezra's proposal," Economic Theory, vol. 26, no. 1, pp. 103-114, 2005. [Online]. Available: http://dx.doi.org/10.1007/s00199-004-0527-z

[22] Z. Han, D. Niyato, W. Saad, T. Başar, and A. Hjorungnes, Game theory in wireless and communication networks: theory, models, and applications. Cambridge University Press, 2012.

[23] L. Chiaraviglio, D. Ciullo, M. Meo, and M. Marsan, "Energy-aware UMTS access networks," in Proc. WPMC, Sep. 2008, pp. 1-8.

[24] A. Bousia, E. Kartsakli, A. Antonopoulos, L. Alonso, and C. Verikoukis, "Game-theoretic infrastructure sharing in multioperator cellular networks," IEEE Transactions on Vehicular Technology, vol. 65, no. 5, pp. 3326-3341, May 2016

[25] B. H., "Spectrum and licensing in the mobile telecommunications market," ITU Regional Workshop on "Competition in Telecommunication Market", May. 2016.

[26] A. Mesodiakaki, F. Adelantado, L. Alonso, M. D. Renzo, and C. Verikoukis, "Energy and spectrum efficient user association in millimeter wave backhaul small cell networks," IEEE Transactions on Vehicular Technology, vol. PP, no. 99, pp. 1-1, 2016.
[27] F. Richter, A. J. Fehske, and G. P. Fettweis, "Energy efficiency aspects of base station deployment strategies for cellular networks," in 2009 IEEE 70th Vehicular Technology Conference Fall, Sep. 2009, pp. 1-5.

[28] 3rd Generation Partnership Project, "Evolved universal terrestrial radio access (E-UTRA); radio frequency (RF) system scenarios," 3GPP TR 36.942, vol. 13.0.0, Rel. 13, Jan. 2016.

[29] R. Hemmecke, M. Kppe, J. Lee, and R. Weismantel, "Nonlinear integer programming," In: Jnger, M., Liebling, T., Naddef, D., Nemhauser, G., Pulleyblank, W., Reinelt, G., Rinaldi, G., Wolsey, L.A. (eds) 50 Years of Integer Programming 19582008. The Early Years and State-of-the-Art Surveys. Springer, 2009.

[30] S. Burer and A. Letchford, "Non-convex mixed-integer nonlinear programming: a survey," Surv. Oper. Res. Manag. Sci. 17(2), pp. 97-106, 2012.

[31] T. H. Cormen, C. E. Leiserson, R. L. Rivest, and C. Stein, Introduction to Algorithms (third edition). MIT Press, 2009.

[32] "International roaming BEREC benchmark data report April-September 2015," Body of European Regulators for Electronic Communications, Mar. 2016.

[33] 3rd Generation Partnership Project, "Further advancements for E-UTRA physical layer aspects," 3GPP TR 36.814, vol. 9.0.0, Rel. 9, Mar. 2010 